curred for a while on every third day, but later became irregular. They were always followed by a high temperature. He was admitted to the hospital there five different times, cach apparent recovery from the fever being followed by a relapse as soon as he returned to duty. During the paroxysms he suffered from very severe headache, muscular pain, nausea and vomiting. He arrived at the Simpson Hospital on Dec. 1I, 1898. Had chilly sensations and headache on the 15th, followed by a rise of temperature. Had three slight paroxysms afterward, accompanied by slight chilly sensations.

Physical Examination: Patient emaciated and anemic; skin yellow, tongue flabby and coated. Heart and lungs normal. Spleen greatly enlarged, reaching nearly to the umbilicus. Liver dulness normal. Bowels constipated. Abdomen tender.

Examination of the Blood: The blood in this case showed numerous pigmented ring forms of the tertian estivo-autumnal parasite and also numerous crescents. The ring forms have already been described under Case 1 .

The erescents were very much more slender and larger than those of the quotidian estivo-autumnal parasites, and contained much more pigment of a more reddish color. The double outline was much less common but the quotidian crescents were more refractive.

In this case the temperature chart is not as typical as the preceding ones, but it is typical of the more chronic form of the tertian estivo-autumnal infection. The paroxysms occur every forty-eight hours approximately, but it will be noted that the range of temperature is not as high and that there seems to be a tendency toward a spontaneous decline of the infection. The chart is curious in that there seems to be a reversal of the ordinary temperature curve, the highest temperature being reached during the initial rise, but even in this chart the temperature curve is on the whole so characteristic that a diagnosis of tertian estivo-autumnal fever could easily be made from an inspection of it.

From the cases of quotidian and tertian estivo-autumnal malarial fever considered it will be seen that no differentiation is possible from a consideration of the clinical symptoms save that in the first named the chill or chilly sensations occur every day, while in the latter they occur every other day. A consideration of the temperature charts, however, shows such a marked difference in the temperature curve that we must admit that we are dealing with two distinct types of infection. The quotidian estivo-autumnal type shows a simple intermittent temperature curve, indistinguishable from a double tertian, while the tertian estivo-autumnal type presents a most peculiar and characteristic temperature curve, entirely different from that shown in any other type of malarial fever, and diagnostic in itself. When to this is added the fact that the tertian form is due to a distinct and easily differentiated parasite, as I have shown, the conclusion is inevitable that there are two varieties of estivo-autumnal fever, the quotidian and tertian, and that each is due to a characteristic parasite, as first shown by Marchiafava and Bignami.

The tertian estivo-autumnal parasite differs from the quotidian parasite in the following particulars:

1. During the hyaline stage: the rings are larger, being from $1 / 8$ to $1 / 4$ the size of the infected corpuscle; the signet-ring shape; the sluggish ameboid motion; the clear-cut and refractive outline; the less wrinkled and lighter green infected corpuscle; the occurrence of only one parasite in the infected corpuscle.

2. During the pigmented stage: larger size, $1 / 2$ of corpuscle; the pigmented ring forms; the continuance of ameboid motion; more sharply defined and refractive, and the granular protoplasm; the finely granular pigment, which is motile; the occurrence of only one parasite in a corpuscle and the lighter colored, seldom crenated infected corpuscle.

3. During the segmenting stage: the occurrence of segmentation outside the corpuscle; the number of segments, ten to fifteen.

4. The larger, more narrow, more deeply pigmented crescents seldom showing a double outline.

5. The cycle of development, forty-eight hours.

In conclusion I desire to express my gratitude to the Surgeon-General of the Army for the opportunities offered me for the study of this subject, and to Col. A. A. Woodhull, Col. A. C. Girard, and Major Charles Richard for their interest in, and encouragement of, the scientific study of disease.

\section{CLINICAL OBSERVATION IN MALARIA AS SEEN IN THE MISSISSIPPI DELTA.*}

\author{
FRANK A. JONES, M.D.
}

Chief of Clinics, Demonstrator of Physical Diagnosis, Instructor Clinical Medicine, etc., Memphis Hospital Medical College. MEMPHIS, TENN.

A distinguished diplomat, scholar and statesman has said: "It is too much the habit of men who have more education than experience, more culture than perception, to desire to mold the policy of the future by avoidance of all cases of trouble in the past." This logic can be well applied to-day in medical research. I come before you not to present anything specially new, but to call your attention briefly to some of the clinical features of malaria as seen in the Mississippi Delta. I shall not attempt a dissertation on the life history of the parasites outside the body, nor to theorize on the relationship which exists between malaria and the much talked-of mosquito. We have been taught much about acute malaria. The biology of the parasites causative of the various forms of malarial fevers has been heralded to us from all quarters of the globe, by men in and out of the malarial districts. With the advent of better laboratory facilities, with higher magnifying lenses, the malarial parasites have been exhaustively studied, while their clinical features have in a great measure been neglected and relegated to an obscure consideration, unfortunately. Malaria in the Mississippi swamps often means chronicity, frequently chronicity without there first having existed acute paroxysms. How often do the Delta physicians see a clinical picture something like this. The patient has neither chill, appetite, nor energy; feels wretched; head and limbs ache; has constant thirst; is constipated; urine is highly colored, scant, and often colored with bile; tongue is thick, flabby and heavily coated; skin is pale and muddy, almost cadaveric; eyes are jaundiced. Physical examination shows liver and spleen to be enormously enlarged. Often the patient will say that he has a "spleen in his side." Pulse is rapid with but little tension. Often on auscultation an anemic murmur will be noticed; temperature is normal. The blood shows anemia, also parasites in various stages of development, especially the estivo-autumnal.

The question naturally arises, Why do all these chronic manifestations occur without there first having existed acute paroxysms? The theory of phagocytosis in all probability explains this relative immunity. The corpuscles being able to cope with this gradual infection, so to speak, the parasites can develop and sporulate without giving rise to paroxysms. I have watched this

* Presented to the Section on Practice of Medicine, at the Fifty. first Annual Meeting of the American Medical Association, held at Atlantic City, N. J., June 5-8, 1900. 
class of patients carefully. Upon sending them to a higher climate, to a climate free from malarial influences, they will in the majority of instances develop paroxysms in a period varying from forty-eight hours to a week.

Why the malaria in this class of patients does not give rise to a paroxysm while in a malarial climate but develops soon after changing to a non-malarial climate remains to be more thoroughly investigated. It is this class of patients who very often become victims of malarial hemoglobinuria.

of late years authorities have been contending over pneumonia, and the course it pursues when malaria is also present. Pneumonia in the Delta or in any highly malarial climate is necessarily more fatal than elsewhere. Pneumonia in the Mississippi Valley runs an irregular course; the crisis is nearly always delayed; the fever is of an irregular type and not the type of a classical text-book pneumonia. Nausea and vomiting are always well-marked symptoms. I have seen malarial paroxysms occur during an attack of pneumonia. A double infection does very often truly exist.

\section{oBESI'TY.}

I have searched the literature carefully but have failed to find anything pertaining to obesity occurring in malarial climates. It is a morbid condition which attracted my attention when I first began to practice in the swamps of Mississippi. It occurs among persons coming from a climate free from malaria to the Delta. During a short residence in the Delta this class take on flesh rapidly; they seem to be immune to malarial infection. They do not have chills, they proclaim the Delta as a health resort on account of their growing fat. They have no chronic manifestations of malaria. It is this class of patients who become victims of furunculosis during the winter months. These are nearly always blondes. I have long since observed that brunettes are more susceptible to malaria than blondes. In all probability the hemoglobin of the brunette is capable of absorbing more poison than that of the blonde. What the pathology of this obesity is remains to be decided. Is it a fatty infiltration, the result of malarial poison, or is it due to stasis in a lymphatic system? It matters not what the pathology may be, the obesity subsides rapidly by changing from a malarial to a non-malarial climate.

\section{PERNICIOUS MALARIA.}

Of the three varieties mentioned in our text-books, the comatose type is most common. Patients are often attacked when there had been no paroxysms or even an initial chill. Temperature generally runs high; it may be subnormal. Prognosis depends on the patient's previous history. If there have been repeated attacks of chills, the prognosis is generally fatal, the system having become so thoroughly surcharged with quinin that the eliminative treatment fails to accomplish anything. On the other hand, we can often effect a cure if the patient's previous health has been good. It is astonishing to note how quickly recovery can sometimes take place by the judicious administration of quinin.

Two-thirds of the population of that portion of the State of Mississippi bordering on the Mississippi River are negroes. The negro constitutes one of the best subjects in which to study the natural course of disease without medication. A" large experience in treating the negro has thoroughly convinced me that he is in a great measure immune to malarial infection. He does not suffer from chronic malaria. Acute paroxysms, when they do occur, are easily cut short by small doses of quinin. I have on numerous occasions seen paroxysms run their course, terminating in spontaneous recovery without any medication. The negro's love for watermelons has been the theme for poets. It is not an uncommon thing for them to have a chill in the early morning hours, pass through the hot and sweating stage by noon, then repair to the patch to partake of a sumptuous feast of hot watermelons which have lain in the sun during the whole day, the repast producing no deleterious effects. Acute nephritis, however, in the negro is quite a common sequel of acute malarial infection. A large clinical experience in conducting an outdoor clinic composed almost entirely of negroes justifies me in making the statement that the negro's kidneys are as vulnerable an organ as his lungs. The negro does not have hemoglobinuria. I have never seen it in the genuine negro; but have in the mulatto. Acute nephritis and glandular fever are common sequelæ of malaria in white children.

\section{UEMOGLOBINURIA.}

The much-discussed, one might say the much-abused, subject of hemoglobinuria has come to be fairly well understood by the majority of the Delta physicians. By careful clinical study, by a large bedside experience, assisted by competent pathologists and microscopists, we have learned that it is the product, the sum total of neglected malaria-a malarial toxemia. In the majority of cases a history of prolonged paroxysms can be obtained. One striking feature of this condition is that it occurs most frequently in its most severe forms with the advent of cold weather. It is more fatal then than when occurring during the summer months. Where it develops in the summer months as a result of previous paroxysms of short duration it runs a much milder course and is more amenable to treatment. Why hemoglobinuria should occur more frequently after the first frost, and why it should prove more fatal then, are questions which demand much consideration. There are just reasons to believe that the parasites remain dormant in the liver, spleen and bone-marrow, a latent malaria. These organs, reacting to the stimulus of a bracing atmosphere become invigorated, sweep the parasites into the peripheral circulation, and take on new life as a consequence of a better circulation. These latent parasites are aroused to activity, toxins are rapidly formed, spending their force on the blood cells, thus producing profound destruction of the corpuscles. The whole system becomes so overwhelmed that it can not react, death ending the scene. Jaundice in this class of patients rapidly becomes profound within a few hours after the chill and after the characteristic discharge has taken place from the kidneys. This jaundice is so pronounced and striking that the late Dr. Warren Stone, of New Orleans, denominated hemoglobinuria as psuedo-yellow fever. We who have seen both diseases have learned that they have many symptoms in common. Pigmentation, both hematogenous and hepatogenous, or ecchymoses on various portions of the body, especially the face, called by the laity "liver spots," are frequently forerunners and forewarners of an approaching attack of hemoglobinuria.

The Delta physicians as well as the laity have learned by experience that the administration of quinin with these conditions present is fraught with danger, frequently precipitating an attack, whereas, if the eliminative course of treatment is pursued, disengorging the liver, kidneys and gastrointestinal tract, followed by a tonic of arsenic, nux vomica and iron, the attack can be prevented. Nausea and vomiting, a tendency to sup- 
pression of the urine and paresis of the bowels, are symptoms which confront the physician and annoy him in treating hemoglobinuria. In those cases in which suppression occurs it appears early in the attack. The nausea and romiting together with suppression, the patient being either in a comatose or a semicomatose condition, present to the physician a clinical picture of uremia. Whether uremia is due to edema of the brain, as advocated by Traube, or whether it is the result of retained toxin, it matters not. We know that where suppression occurs in hemoglobinuria the patient in nearly every instance succumbs, it matters not how heroic nor how various our therapeutic measures have been. If we accept the physiological teaching that the liver creates urea and that it is eliminated by the kidneys, there is just reason to suppose that this urea-producing function of the liver, by reason of its being congested and engorged, is interfered with; the kidneys are called on not only to eliminate the malarial toxin but also the toxins of imperfect liver functions; hence the suppression, hence the coma. Anuria of several days' duration is not an uncommon occurrence in hemoglobinuria. I have recorded one patient who lived nine days. When I saw him he had had several discharges from the kidneys. He had been living in the swamps of Arkansas and had had numerous paroxyms during a period of several years. The liver and spleen were enormously enlarged. He was profoundly anemic; had no nausea or romiting, and was in a semi-comatose condition, with temperature subnormal during the whole time. He died on the ninth day after my first visit. He had no convulsions, dissolution taking place by a gradual process. I believe that a true splenic leukemia - which has been so clearly and concisely described by Dr. Osler ${ }^{1}$-much more frequently exists than is generally supposed. What the physicians of the swamps of Arkansas, Louisiana and Mississippi need are better facilities for making blood analyses.

I wish to call attention to a condition which $I$ have frequently observed in hemoglobinuria, and I have seen no mention of it. I refer to paresis of the bowels. Text-books tell us that diarrhea is often present in hemoglobinuria. This has not been my experience. Diarrhea is the exception and not the rule. It seems that with this condition diarrhea in a measure might prove salutary. Very often just as suppression occurs the abdomen becomes distended with gas. The medicine which you have given your patient with a view of arousing the liver and kidneys to action has had no effect. Death takes place, both by suppression and paresis of the bowels. It seems that the same conditions which have produced the suppression have also acted as causative factors of the paresis. Iret me admonish you, gentlemen, who are making scientific research in malaria not to journey to Rome to study the Pontine fevers or to South Africa to investigate the black-water fever, but come South to the swamps of Arkansas, Mississippi and Louisiana, where we have malaria in abundance and in all of its forms.

Sanitary Substitute for the Individual Communion Cup.-The Lancet suggests the revival of the ancient custom of dipping the bread in the wine or "intinction," instead of the use of the common chalice at the Communion. This is the method still followed in the Eastern Church, and was generally practised until the twelfth century. The editorial earnestly urges ecclesiastical authorities to consider the matter and live up to the traditions of the Church as a leader in sanitary matters. Individual communion cups are not practicable. but intinction has much to recommend it.

1. Ameritan Journal of the Medical sciences.

\section{HYPERTROPHY OF PHARYNGEAL TONSIL.}

ITS ANATOMY AND PHISIOLOGY.*

NORVAL H. PIERCE, M.D.

CHICAGO.

The pharyngeal tonsil lies above a line drawn from about the middle of the Eustachian cushions in the postnasal space, and is developed at a very early age, probably coincident with the faucial tonsils. Its ultimate nature is as much unknown as is the ultimate nature of the tonsils. Whether it is an evolutionary vestige or a gland which has still a function is unknown at the present time. Embryologically, it develops with the pituitary body and the pineal gland, and probably there is a relationship existing between these three bodies. It is composed of lymphoid tissue, the mass of the tissue being round cells indistinguishable from embryological connective tissue and lymph nodes, which are in turn indistinguishable from the solitary follicles of the intestine. These, together with the blood-vessels and nerves-for there are nerves in the pharyngeal tonsil and adenoids-are held together by a reticulum of connective tissue, which has a great deal to do with the involution of the organ after hypertrophy. Each of the lymph nodes or solitary follicles is encased in a bag of connective tissue. This connective tissue, at an early date, is embryonal in character and goes onward to adult growth, ripens rather early, and in due course of time matures, and, following the law of connective tissue, contracts, thus squeezing the lymph nodes, shutting off the blood-supply to them, and by pressure atrophy, we may say, producing their shrinkage. This body is covered by ciliated columnar epithelium, which differs from the epithelium of the mesopharynx. However, in the majority of growths that I have examined, I have found as much squamous or cuboidal as columnar epithelium. This transition from the columnar to the cuboidal variety is due to pressure. Adenoids are hyperplasia of this normal tissue. They vary, of course, in size, in consistency, and in color. The surface may be coarsely granular, furrowed more or less, or smooth. The smooth variety very frequently leads us astray in post-rhinoscopic diagnosis, because it forms simply a smooth layer, thus foreshortening the nasal space, and this is difficult to judge in the mirror.

I have under the microscope four specimens which show the various interesting points in adenoids, one particularly which shows the peculiar, fan-shaped distribution of the blood-vessels, which are surrounded by connective tissue; this apparently holds the blood-vessels open, thus increasing the blood-supply to the gland. In this way we may account for hyperplasia in some of these bodies. The hyperplasia, however, is most often caused by a succession of attacks of acute inflammation, just as hyperplasia of the faucial tonsil is caused by hypertrophy.

The infectious diseases, such as measles, scarlet fever, etc., play a very active part in the causation of this condition. Then, too, of the acute inflammations of the gland itself, we have the lacunar variety of inflammation, and the inflammations due to the streptococcus infections, which are very frequently the cause of acute hyperplasia.

Their dependence on tuberculosis is a most interesting and important question, and it is still unsettled. Dansac, in an interesting article which appeared in

* Read in a Symposium on Hypertrophy of the Pharyngeal Tonsil, before the Chicago Climatological and Laryngological Association, July 5. 1900 . 